\title{
THE EFFECTS OF PALMVITEE ON HUMAN NASAL SEPTAL CHONDROCYTES CULTURE EXPANSION AND CARTILAGE RECONSTRUCTION
}

\author{
B.H.I. Ruszymah*, K.H. Chua, A.L. Mazlyzam \\ Department of Physiology, Medical Faculty of Universiti Kebangsaan \\ Malaysia, Jalan Raja Muda Abdul Aziz, 50300 Kuala Lumpur, Malaysia
}

\section{B.S. Aminuddin}

Tissue Engineering Laboratory, Hospital Universiti Kebangsaan Malaysia, Jalan Yaacob Latif, 56000 Cheras, Kuala Lumpur, Malaysia

\author{
A. Gapor \\ Malaysia Palm Oil Board, No 6, Persiaran Institusi, Bandar Baru \\ Bangi 43000 Kajang Selangor, Malaysia \\ H. Fuzina \\ Animal Unit, Institute for Medical Research, Jalan Pahang, \\ 50588 Kuala Lumpur, Malaysia.
}

Received 01 August 2005,

\begin{abstract}
Palmvitee may act as a beneficial supplement to cartilage tissue engineering which is currently still has certain limitation on chondrocyte expansion and dedifferentiation. Our objectives in this study were to evaluate the effects of Palmvitee on the growth kinetic and phenotype gene expression of human nasal septal chondrocytes in monolayer expansion as well as cartilage reconstruction via tissue engineering technology. Human chondrocytes were cultured in mediums containing various Palmvitee concentrations. Among the different test groups, the medium containing Palmvitee at $3 \mu \mathrm{g} / \mathrm{ml}$ supported the highest chondrocyte growth rate. The gene expression in monolayer chondrocytes culture supplemented with $3 \mu \mathrm{g} / \mathrm{ml}$ Palmvitee demonstrated similar results as in control. Cultured chondrocytes from the medium added with 3 $\mu \mathrm{g} / \mathrm{ml}$ Palmvitee were then mixed with Pluronic F127 for cartilage reconstruction in nude mice model. Palmvitee supplementation supported the engineered cartilage development as shown in the histological and gene expression analysis on engineered tissue after 8 weeks of implantation. Therefore, Palmvitee at $3 \mu \mathrm{g} / \mathrm{ml}$ is beneficial for the human nasal septal chondrocytes monolayer expansion and cartilage engineering.
\end{abstract}

*Corresponding author e-mail: ruszy@medic.ukm.my 


\section{INTRODUCTION}

Vitamin E is a group of lipid-soluble, potent chain breaking antioxidant. The molecules can be subgrouped into tocopherols and tocotrienols [1, 2, 21, 23, 24, 27]. Tocopherols are commonly found in a wide variety of food such as nuts and common vegetable oils; whereas tocotrienols are relatively rare and can be found only in a few specific vegetable oils such as palm oil [3, 4, 20]. Tocopherols and tocotrienols are both potent antioxidants. However, the antitumor activity is not depending on their antioxidant activity. Majority of studies have shown that tocotrienols provide greater antitumor activity compared to tocopherols [4, 5, 6, 21, 22, 25 - 27]. Previous studies showed that these compounds inhibit growth and tumor development by modulating multiple intracellular signaling pathways, including inhibiting protein kinase $C$, adenylate cyclase and cyclic AMP - dependent protein activation that involves in mitogenesis and apoptosis $[4,5,27]$. Tocotrienols and tocopherols also have antioxidant activity that reacts on reactive oxygen species that is known as free radicals [6 - 9, 27]. Their chain breaking properties neutralize peroxyl and alkoxyl radicals generated during lipid peroxidation. Membrane lipid peroxidation is known to modify and inactivate cellular components, which can cause damaging effects on crucial cellular factors leading to cell damages.

Tissue engineering is a new field with a vision to re-engineer and to reconstruct biological substitute that can replace the loss of tissues or organs through trauma or diseases. Engineered organs or tissues need to be functional physiologically as the original organ or tissues [10]. Tissue engineering has given hope to millions of people who are waiting for cartilage replacement for repairing the defect tissues of the nose, ear, trachea and joints [11, 12]. Chondrocytes are cells that produce the extra cellular matrix for cartilage, especially collagen type II that acts as the important building block of cartilage. Human nasal septal cartilage is a good candidate as a starting material for cartilage tissue engineering since it is high in cell density and provides chondrocytes with excellent proliferation capacity. Furthermore, it can be isolated from a broad range of human age $[13,14]$. However, this approach is currently limited by the generation of dedifferentiated cultured chondrocytes in in vitro monolayer system. The in vitro culture is very important in increasing the number of the cells harvest from the sample since there is only small number of cells that can be obtained. Increasing the cell numbers is very crucial since millions of cells are needed for the tissues or organs reconstruction processes. Previous studies have shown growth factors; for instance, fibroblast growth factors (bFGF) can increase the number of chondrocytes in the monolayer culture $[15,16]$. However, cultured chondrocytes produce lower cell viability, decreasing growth rate and increasing doubling time with increasing number of passages. The dedifferentiated cultured chondrocytes were not able to maintain the expression of type II collagen in long term in vitro monolayer culture system ${ }^{17,18}$. No study has been done to determine the effects of Palmvitee on human chondrocytes monolayer culture and cartilage tissue engineering. In this study, our objectives were to evaluate the effects of Palmvitee on the growth kinetic and phenotype gene expression of human nasal septal chondrocytes in monolayer expansion as well as the cartilage reconstruction via tissue engineering technology.

\section{MATERIALS AND METHODS}

\subsection{Human nasal septal harvest}

Nasal septal cartilages were obtained from 6 consented patients after elective septoplasty. These specimens would usually be discarded and their uses in this study have been approved by the Research and Ethical Committee of Medical Faculty, Universiti Kebangsaan Malaysia, 
Malaysia. Removed cartilage was placed in sterile normal saline at $4{ }^{\circ} \mathrm{C}$ and transported to the Tissue Engineering Laboratory, Hospital Universiti Kebangsaan Malaysia.

\subsection{Nasal septal chondrocyte isolation}

Each cartilage was stripped from their perichondrium, minced into small pieces $\left(1 \mathrm{~mm}^{3}\right)$, washed with phosphate buffer saline (PBS; pH 7.2; Gibco, Grand Island, NY) containing 100 $\mathrm{U} / \mathrm{mL}$ of penicillin, $100 \mu \mathrm{g} / \mathrm{mL}$ of streptomycin and $0.25 \mu \mathrm{g} / \mathrm{mL}$ of amphotericin B (Gibco, Grand Island, NY) and digested with $0.6 \%$ collagenase II (Gibco, Grand Island, NY) in an orbital incubator (Stuart Scientific, Redhill, UK) at $37^{\circ} \mathrm{C}$ for 12 hours. Isolated chondrocyte suspensions were then centrifuged $\left(37^{\circ} \mathrm{C}\right)$ at $6,000 \mathrm{rpm}$ for 5 minutes. The resulting cell pellet was washed twice with PBS to remove remaining digestive enzyme. After the final centrifugation, the cell pellet was re-suspended in PBS for total the cell count with hemocytometer (Weber Scientific International Ltd. Middlx, England) and cell viability was determined using trypan blue dye via cell exclusion test (Gibco, Grand Island, NY).

\subsection{Chondrocytes in vitro culture}

Freshly isolated chondrocytes with 10,000 cells $/ \mathrm{cm}^{2}$ density were then seeded in a 6 -well tissue culture plate (Falcon, Franklin Lakes, NJ) as the primary culture (P0) in medium consisting of equal volume of Ham's F12 medium and Dulbecco's Modified Eagle Medium (F12:DMEM; Gibco). The medium was nourished with $10 \%$ fetal bovine serum (FBS; Gibco, Grand Island, NY), $200 \mathrm{mM}$ L-glutamine (Gibco, Grand Island, NY), $100 \mathrm{U} / \mathrm{mL}$ of penicillin (Gibco, Grand Island, NY), $100 \mu \mathrm{g} / \mathrm{mL}$ of streptomycin (Gibco, Grand Island, NY), $0.25 \mu \mathrm{g} / \mathrm{mL}$ of amphotericin B (Gibco, Grand Island, NY) and $50 \mu \mathrm{g} / \mathrm{mL}$ of ascorbic acid (Sigma, St. Louis. MO). The medium was further supplemented with Palmvitee [Malaysia Palm Oil Board (MPOB)] at various concentration; $0 \mu \mathrm{g} / \mathrm{ml}$ (control), $3 \mu \mathrm{g} / \mathrm{ml}, 6 \mu \mathrm{g} / \mathrm{ml}, 10.5 \mu \mathrm{g} / \mathrm{ml}$ and 15 $\mu \mathrm{g} / \mathrm{ml}$. All cultures were maintained in $5 \% \mathrm{CO}_{2}$ incubator (Jouan, Duguay Trouin, $\mathrm{SH}$ ) at $37^{\circ} \mathrm{C}$ with the culture medium changed twice a week. When the primary culture (P0) reached confluence, it was trypsinized with $0.05 \%$ trypsin-EDTA (Gibco, Grand Island, NY). The harvested cultured chondrocytes were washed three times in PBS (Gibco, Grand Island, NY). The resulted cell pellet was then re-suspended in $10 \mathrm{~mL}$ of PBS for total cell count with hemocytometer (Weber Scientific International Ltd.) and cell viability was determined by trypan blue dye exclusion test. Cultured chondrocytes were then sub-cultured twice (P1 and P2) into a new well of 6-well tissue culture plate (Falcon) with the same type of medium and culture environment. The total cell number and the cell viability were recorded at every passage.

\subsection{Chondrocytes monolayer culture characterization}

Cell morphological features in the culture were examined everyday by using an inverted light microscope (Olympus, Shinjuku-ku, Tokyo). Viability of cultured chondrocytes in each Palmvitee concentration at every passage (P0, P1 and P2) was calculated as percentage of living chondrocyte cells from each cell culture at each passage. Growth rate of cultured chondrocytes in each Palmvitee concentration at every passage (P0, P1 and P2) was calculated as average increase of chondrocyte number in one day $/ \mathrm{cm}^{2}$.

\subsection{Total RNA extraction}

Total RNA from primary culture (P0) and each passage (P1 and $\mathrm{P} 2$ ) were extracted for phenotype gene expression analysis. Total RNA was extracted using TRI Reagent (Molecular Research Center, Cincinnati, $\mathrm{OH}$ ) according to the manufacturer's instruction. Polyacryl Carrier 
$[17,18]$ (Molecular Research Center, USA) was added in each extraction to precipitate the total RNA. RNA pellet was then washed with $75 \%$ ethanol and air-dried before dissolved in RNase and DNase free distilled water (Invitrogen, Carlsbad, CA). The yield and purity of the isolated RNA were determined by spectrophotometer (Bio-Rad, Hercules, CA). Total RNA were stored at $-80^{\circ} \mathrm{C}$ immediately after extraction.

\subsection{One step reverse transcriptase-polymerase chain reaction (RT-PCR)}

Chondrocyte phenotype expression of type I and type II collagen genes were evaluated by one step reverse transcriptase-polymerase chain reaction (One Step RT-PCR; Invitrogen, Carlsbad, CA). Human $\beta$-actin gene expression was used as control. The specific sense and antisense primers used for the reaction were designed from listed NIH GenBank database and had the following sequences: type I collagen, 5'-AGGCTTCCAAGGTCCCCCTGGTG-3' and 5'AGCACCAGTAGCACCATCATTTC-3';

type II collagen, 5'-CTGGCAAAGATGGTGAGACAGGTG-3' and 5'-GACCA- TCAGTGC CAGGAGTGC-3'; $\beta$-actin, 5'-CCGGCTTCGCGGGCGACG-3' and 5'-TCCCGGCC AGCCAGGTCC-3'. One Step RT-PCR reaction mix was prepared according to the manufacturer's instruction. Each reaction consisted of $100 \mathrm{ng}$ total RNA and $10 \mathrm{pmol}$ of each sense and antisense primers. One step RT-PCR was performed in a 9700 thermal cycler (Perkin Elmer, Norwalk, CT) with reaction profile of; cDNA synthesis for $30 \mathrm{~min}$ at $50^{\circ} \mathrm{C}$; predenaturation for $2 \mathrm{~min}$ at $94^{\circ} \mathrm{C}$; PCR amplification for 38 cycles with $30 \mathrm{sec}$ at $94^{\circ} \mathrm{C}, 30 \mathrm{sec}$ at $60^{\circ} \mathrm{C}$ and $1 \mathrm{~min}$ at $72^{\circ} \mathrm{C}$. This series of cycles was followed by a final extension of $72^{\circ} \mathrm{C}$ for 2 min. The PCR products were electrophoresed on a $1.5 \%$ agarose gel (Invitrogen, Carlsbad, CA) stained with ethidium bromide (Sigma, St. Loius, MO) and visualized by using UV transillumination (Vilber Lourmat, Marne La Vallee, France).

\subsection{Large-scale chondrocyte culture for cartilage tissue engineering}

Chondrocyte culture medium supplemented with $3 \mu \mathrm{g} / \mathrm{ml}$ of Palmvitee was used for large-scale chondrocyte culture. Chondrocytes were plated in $175 \mathrm{~cm}^{2}$ culture flasks (Falcon, Franklin Lakes, NJ) at a density of 10,000 cells $/ \mathrm{cm}^{2}$. When the culture reached confluence, the chondrocytes were trypsinized using $0.05 \%$ trypsin-EDTA (Gibco). The culture-expanded chondrocytes were then suspended in a hydrogel; Pluronuic F127 for engineered cartilage formation.

\subsection{Cartilage tissue engineering}

Cultured chondrocytes were suspended into a cold $\left(4^{\circ} \mathrm{C}\right) 30 \%$ (wt/vol) co-polymer of polyethylene oxide and polypropylene oxide, Pluronic F-127 (BASF, Mount Olive, NJ) solution at a cell density of $3 \times 10^{7}$ cells $/ \mathrm{mL}$. The admixer was then injected subcutaneously at the dorsal part of the 8-week old nude mice under general anesthesia (Ketamine, Xylazine and Zoletil). The engineered tissues were harvested after 8 weeks of in vivo implantation. The nude mice were then sacrificed and engineered tissues were harvested and carefully dissected from surrounding soft tissue.

\subsection{Engineered cartilage evaluation}

After the engineered tissue was removed from the nude mice, it was divided equally into two. One part was fixed in 10\% phosphate-buffered formalin (Fisher Scientific, Fair Lawn, NJ), embedded in a paraffin block and then sectioned for histological analysis. Slides sections were 
stained with hematoxylin \& eosin (H\&E staining) to evaluate cell morphology and tissue organization whereas Safranin O staining was used to evaluate the cartilage proteoglycans production. The other half of the excised tissue was digested with collagenase and total RNA was extracted from the isolated cells using the above mentioned techniques. Phenotype gene expression of the cells isolated from the engineered cartilage was evaluated by one-step RTPCR technique.

As a comparison, large scale culture expansion was also done by using the culture medium without Palmvitee supplementation.

\subsection{Statistical analysis}

Data for chondrocyte viability and growth rate in each Palmvitee concentration at every passage (P0, P1 and P2) were collected from all samples. Values were presented as mean \pm standard error of mean (SEM). Student's $t$ test was used to compare the data between groups and differences at the $5 \%$ level were considered significant.

\section{RESULTS}

\subsection{Morphological features of human nasal septal chondrocytes in monolayer culture}
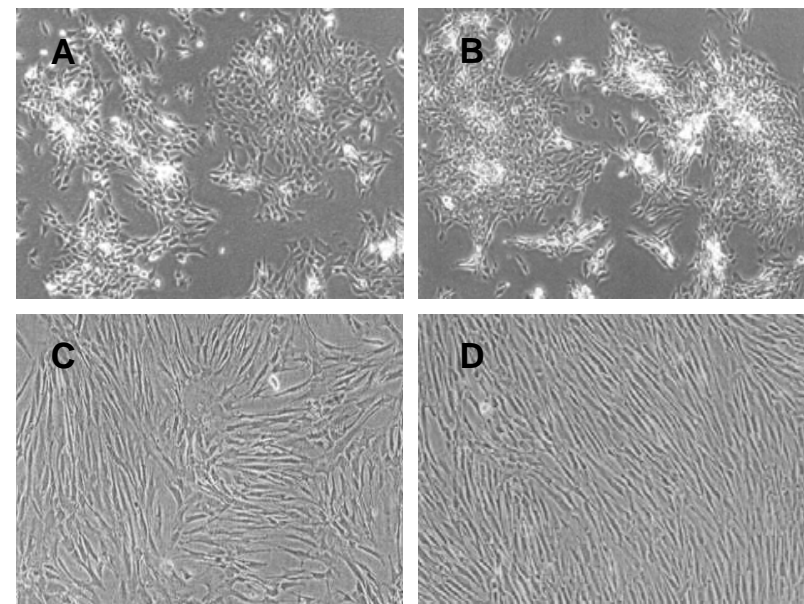

Fig. 1: $\quad$ Morphological features of human nasal septal chondrocytes at primary culture (A \& B) and passage $2(\boldsymbol{C} \& \mathbf{D})$. Chondrocytes were cultured in medium without Palmvitee supplementation (A \& C) and medium added with $3 \mu \mathrm{g} / \mathrm{ml}$ Palmvitee (B \& D). Chondrocytes at primary culture appeared uniform in size with polygonal shape and distributed as islet of growth (A \& B). Chondrocytes at passage 2 were elongated in shape. Palmvitee supplementation supported higher chondrocyte density at primary culture and passage 2 (B \& D). Magnification $=40 X$

Freshly isolated chondrocytes started to adhere onto the tissue culture plate on the second day of primary culture. Cell multiplication started on the third day and became prominent after 1 week of culture. Chondrocytes appeared uniform in size with polygonal shape and distributed as islet of growth (Fig. 1). Chondrocytes cultured in medium supplemented with $3 \mu \mathrm{g} / \mathrm{ml}$ Palmvitee exhibited higher chondrocyte density (Fig. 1B) compared to chondrocytes cultured in medium 
without Palmvitee supplementation (Fig. 1A). Chondrocytes at primary culture (P0) in all experiment groups became confluent within 3 weeks and remained polygonal in shape. As the cultured chondrocytes were passaged, the shape tends to become elongated; grew slower and the cultures usually take longer time to reach confluence in all the experiment groups (Fig. 2). Palmvitee supplementation at $3 \mu \mathrm{g} / \mathrm{ml}$ concentration (Fig. 2B) continued to support a higher chondrocytes density compared to other groups at passage 2 .

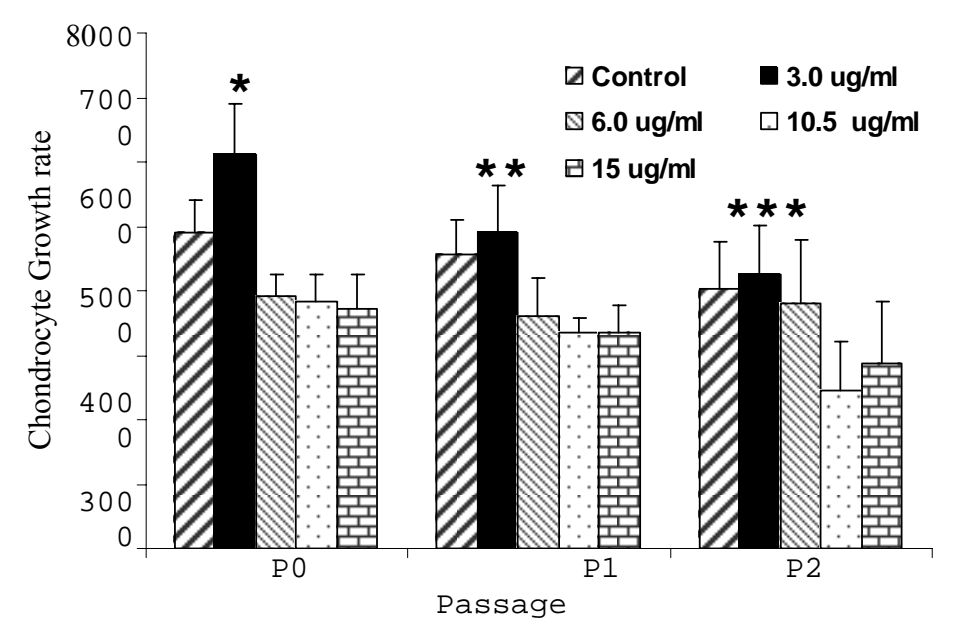

Fig. 2: Growth rate (average increase in cell number per day/ $\mathrm{cm}^{2}$ ) of human nasal septal chondrocytes cultured in medium supplemented with various Palmvitee concentrations at primary culture (P0), passage 1 (P1) and passage 2 (P2). All values were showed as mean $\pm \operatorname{SEM}(n=6)$.

*Chondrocyte growth rate in medium added with $3 \mu \mathrm{g} / \mathrm{ml}$ Palmvitee was significant higher than control $(0 \mu \mathrm{g} / \mathrm{ml}), 6 \mu \mathrm{g} / \mathrm{ml}, 10.5 \mu \mathrm{g} / \mathrm{ml}$ and $15 \mu \mathrm{g} / \mathrm{ml}$ Palmvitee at primary culture (P0), $(p<0.05)$.

**Chondrocyte growth rate in medium added with $3 \mu \mathrm{g} / \mathrm{ml}$ Palmvitee was significant higher than $6 \mu \mathrm{g} / \mathrm{ml}, 10.5 \mu \mathrm{g} / \mathrm{ml}$ and $15 \mu \mathrm{g} / \mathrm{ml}$ Palmvitee at passage 1 (P1), ( $<<0.05)$.

***Chondrocyte growth rate in medium added with $3 \mu \mathrm{g} / \mathrm{ml}$ Palmvitee was significant higher than $10.5 \mu \mathrm{g} / \mathrm{ml}$ and $15 \mu \mathrm{g} / \mathrm{ml}$ Palmvitee at passage $2(P 2),(p<0.05)$.

\subsection{Chondrocyte growth kinetics at multiple passages}

Human nasal septal chondrocytes growth rate (average increase in cell number per day $/ \mathrm{cm}^{2}$ ) was the highest in medium supplemented with $3 \mu \mathrm{g} / \mathrm{ml}$ Palmvitee at primary culture (P0), passage 1 (P1) and passage 2 (P2) compared to other experiment groups (Fig. 2). Chondrocytes cultured in medium supplemented with $3 \mu \mathrm{g} / \mathrm{ml}$ Palmvitee scored $6120 \pm 777$ cells $/$ day $/ \mathrm{cm}^{2}$ growth rates at primary culture. This value was significantly higher than all the other experiment groups $(\mathrm{p}<$ 0.05; Fig. 2). Chondrocyte growth rate reduced to $3920 \pm 334 \mathrm{cells} / \mathrm{day} / \mathrm{cm}^{2}$ when the Palmvitee concentration was increased to $6 \mu \mathrm{g} / \mathrm{ml}$ at primary culture (Fig. 2). Further increment in Palmvitee concentration to $10.5 \mu \mathrm{g} / \mathrm{ml}$ and $15 \mu \mathrm{g} / \mathrm{ml}$ only caused minor decreases in chondrocyte growth rate at primary culture (Fig. 2). Chondrocyte cultured in medium supplemented with $3 \mu \mathrm{g} / \mathrm{ml}$ Palmvitee scored $4894 \pm 731$ cells $/$ day $/ \mathrm{cm}^{2}$ growth rate at passage 1 (P1). This value was only slightly higher than the control $(0 \mu \mathrm{g} / \mathrm{ml}$ palmvitee $)$, but was significantly higher compared to the experiment groups supplemented with $6 \mu \mathrm{g} / \mathrm{ml}, 10.5 \mu \mathrm{g} / \mathrm{ml}$ and $15 \mu \mathrm{g} / \mathrm{ml}$ Palmvitee at $\mathrm{P} 1$ ( $\mathrm{p}<0.05$; Fig. 2). At passage 2, chondrocytes cultured in medium supplemented with $3 \mu \mathrm{g} / \mathrm{ml}$ Palmvitee scored $4266 \pm 756$ cells/day $/ \mathrm{cm}^{2}$ growth rate (Fig. 2). 
Chondrocyte growth rate in the medium added with $3 \mu \mathrm{g} / \mathrm{ml}$ Palmvitee was significantly higher than the medium added with $10.5 \mu \mathrm{g} / \mathrm{ml}$ and $15 \mu \mathrm{g} / \mathrm{ml}$ Palmvitee at passage $2(\mathrm{p}<0.05$; Fig. 2).

Chondrocytes cultured in medium supplemented with various Palmvitee concentrations scored cell viability ranging from $88 \%$ to $97 \%$ in multiple passages. By using the Students' $t$ test, there was no significant difference when these values between the groups were compared.

\subsection{One step RT-PCR analysis}

Freshly isolated human nasal septum chondrocytes only expressed type II collagen but not type I collagen (Fig. 3). During monolayer expansion, cultured chondrocytes demonstrated similar gene expression pattern regardless of the Palmvitee concentration supplemented into the medium. At primary culture, the cultured chondrocytes from all experiment groups expressed both type I and type II collagen gene at the same intensity (P0, Fig. 3). At passage 1, the expression on the type II collagen was less intense (showed by a faint band in the gel photo) compared to the expression of the type I collagen gene (P1, Fig. 3). The cultured chondrocytes at passage 2 from all the different experiment groups showed a negative expression on type II collagen gene; however, the type I collagen gene was continually expressed (P2, Fig. 3).

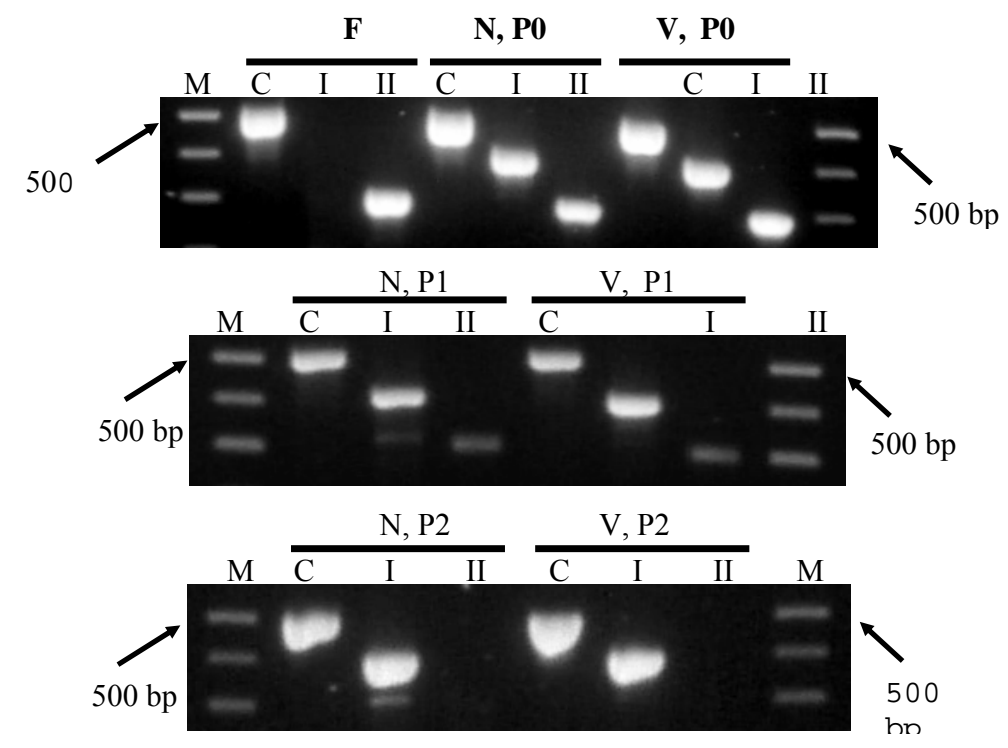

Fig. 3: One step reverse transcriptase-polymerase chain reaction analysis on the expression of type I and type II collagen gene from freshly isolated chondrocytes (F) and cultured chondrocytes at primary culture (P0), passage 1 (P1) and passage 2 (P2). Chondrocytes cultured in medium without Palmvitee supplementation (N) and with $3 \mu \mathrm{g} / \mathrm{ml}$ Palmvitee supplementation (V). Freshly isolated chondrocytes only expressed type II collagen but not type I collagen (top gel photo). Cultured chondrocytes expressed both type I and type II collagen at P0 (top gel photo). At P1, expression of type II collagen gene was reduced (middle gel photo) and cultured chondrocytes at P2 showed negative expression of type II collagen gene (lowest gel photo). Expression of $\beta$-actin gene was acted as a control. Lane M: 100 bp DNA marker. Lane $C$ : $\beta$-actin gene fragment (495 bp). Lane I: Type I collagen gene fragment (396 bp). Lane II: Type II collagen gene fragment (294 bp).

\subsection{Cartilage tissue engineering and evaluation}


Since the medium supplemented with $3 \mu \mathrm{g} / \mathrm{ml}$ Palmvitee demonstrated the best chondrocyte growth rate, this medium was then used in large-scale chondrocytes culture for cartilage reconstruction via tissue engineering technology. The medium without Palmvitee supplementation was used as control for in vivo cartilage reformation.
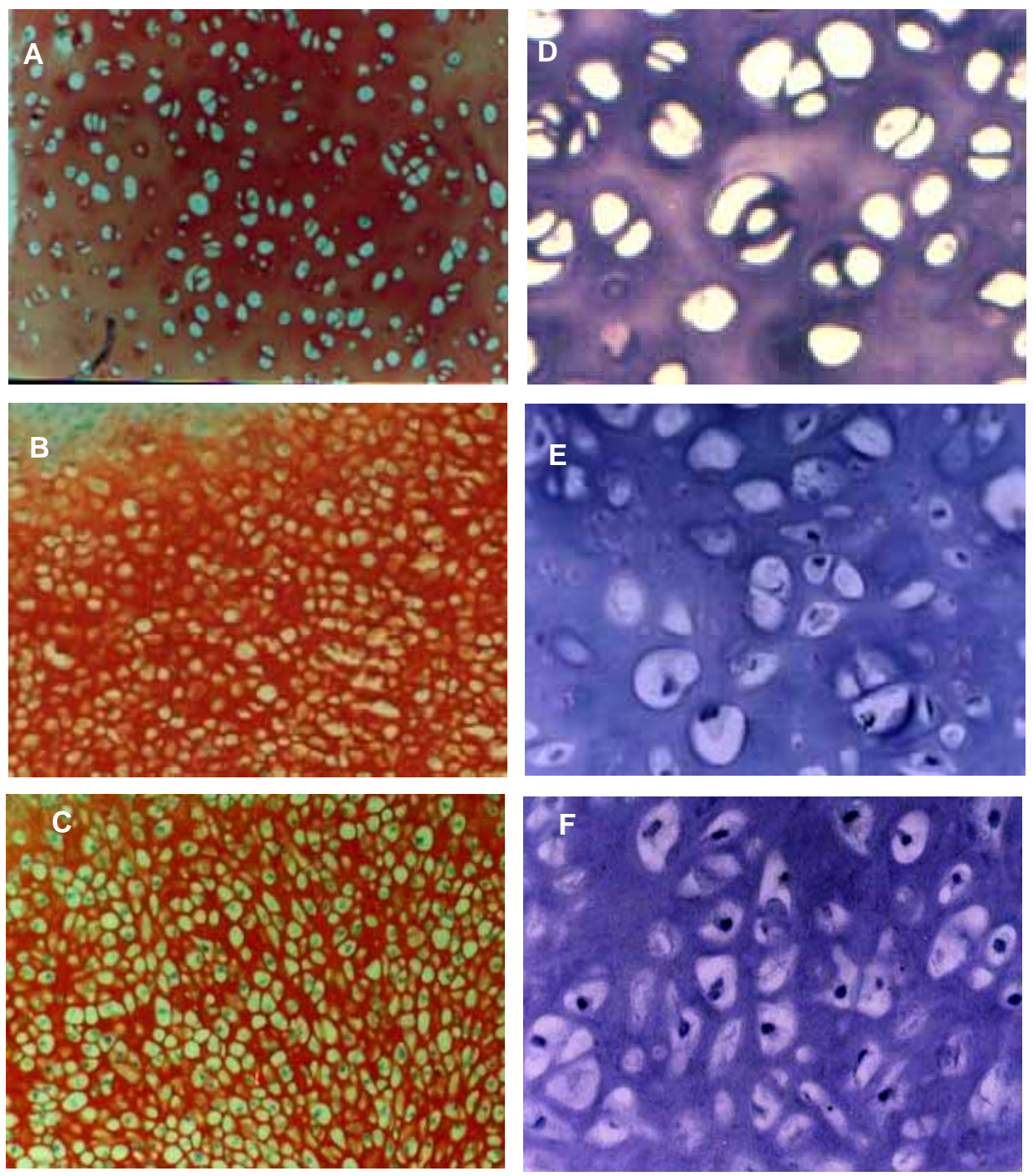

Fig. 4: Histological evaluation on the native cartilage and reconstructed cartilages removed from nude mice after 8 weeks of in vivo implantation. Safranin $O$ staining $(\boldsymbol{A}, \boldsymbol{B} \& \boldsymbol{C})$, magnification $=40 X$. Strong positive orange-red staining of Safranin $O$ on human native nasal septal cartilage (A), reconstructed cartilage from culture medium without Palmvitee supplementation (B) and reconstructed cartilage with $3 \mu \mathrm{g} / \mathrm{ml}$ Palmvitee supplementation $(\boldsymbol{C})$ correlating with abundant proteoglycan production. Hematoxylin $\&$ eosin staining $(\boldsymbol{D}, \boldsymbol{E} \& \boldsymbol{F})$, magnification $=200 X$. Hematoxylin \& eosin staining demonstrated human native nasal septum cartilage (D), reconstructed cartilage from culture medium without Palmvitee supplementation (E) and reconstructed cartilage with $3 \mu \mathrm{g} / \mathrm{ml}$ Palmvitee supplementation $(\boldsymbol{F})$ were consisted of evenly spaced lacunae cells embedded in a basophilic matrix.

After 8 weeks of in vivo development of the reconstructed cartilages in nude mice model, Safranin O staining on the histological sections of reconstructed cartilages from the $3 \mu \mathrm{g} / \mathrm{ml}$ 
Palmvitee supplemented culture medium (Fig. 4C) as well as the control (medium without Palmvitee supplementation; Fig. 4B) exhibited a strong positive orange-red staining comparable to the human native nasal septal cartilage sections (Fig. 4A). The positive orange-red staining from Safranin $\mathrm{O}$ denotes abundant cartilage proteoglycans production in the tissues. Hematoxylin \& Eosin staining on the reconstructed cartilages from the cultured medium without Palmvitee supplementation (Fig. 4E) and from the reconstructed cartilages with $3 \mu \mathrm{g} / \mathrm{ml}$ Palmvitee supplementation (Fig. 4F) showed the tissue sections were consisted of evenly spaced lacunae cells embedded in a basophilic matrix. These histological features were similar to the human native nasal septum cartilage (Fig. 4D). The histological staining showed that the reconstructed cartilage was higher in cell density compared to the native cartilage.

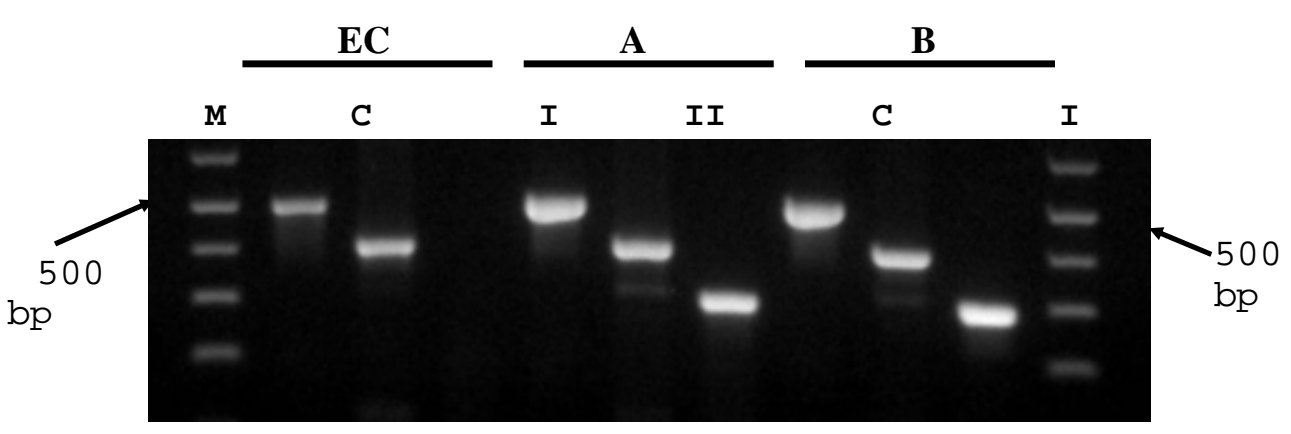

Fig. 5: $\quad$ One step reverse transcriptase-polymerase chain reaction analysis on the expression of type I and type II collagen gene from the large scale expanded chondrocytes in monolayer culture (EC), reconstructed cartilage from culture medium without Palmvitee supplementation (A) and reconstructed cartilage from culture medium added with $3 \mu \mathrm{g} / \mathrm{ml}$ Palmvitee (B). Large scale expanded chondrocytes expressed only type I collagen but not type II collagen. Both reconstructed cartilages; $\boldsymbol{A}$ and $\boldsymbol{B}$ demonstrated re-expression of type II collagen gene. Expression of $\beta$-actin gene was acted as a control. M: 100 bp DNA marker. Lane $C$ : $\beta$-actin gene fragment (495 bp). Lane I: Type I collagen gene fragment (396 bp). Lane II: Type II collagen gene fragment (294 bp).

One step reverse transcriptase-polymerase chain reaction (RT-PCR) analysis on the total RNA extracted from large scale expanded chondrocytes showed the cultured chondrocytes only expressed type I collagen but not type II collagen (Fig. 5). After 8 weeks of in vivo development of the reconstructed cartilages, both reconstructed cartilages from the culture medium with $3 \mu \mathrm{g} / \mathrm{ml}$ Palmvitee supplementation and the control (medium without Palmvitee supplementation) demonstrated a high re-expression of type II collagen gene (Fig. 5). Type I collagen gene was also co-expressed in the tissues.

\section{DISCUSSION}

Our current data presents the first report of the effects of Palmvitee on the human nasal septal chondrocyte monolayer expansion and cartilage reconstruction. The supplementation of Palmvitee up to $15 \mu \mathrm{g} / \mathrm{ml}$ in mediums did not alter the morphological features of cultured chondrocytes in monolayer expansion. The change on chondrocyte morphology from polygonal to elongated shape was due to the modification on the cell cytoskeleton in the monolayer culture [28].

Supplementation of Palmvitee at $3 \mu \mathrm{g} / \mathrm{ml}$ to the culture medium increased chondrocytes proliferation in multiple passages compared to the control medium without additional Palmvitee. This result shows that Palmvitee at $3 \mu \mathrm{g} / \mathrm{ml}$ concentration has small mitogenic effect 
on human nasal septum chondrocytes. The antioxidant activity of Palmvitee that reduce the cell injury by free radicals may be the possible reason for the minor mitogenic property of Palmvitee on chondrocyte expansion [6 - 9, 27]. The addition of Palmvitee at $3 \mu \mathrm{g} / \mathrm{ml}$ into the culture medium was shown to be beneficial for the human nasal septal chondrocytes monolayer expansion since it can increase the amount of chondrocytes for cartilage reconstruction. However, supplementation of Palmvitee at higher concentration than $3 \mu \mathrm{g} / \mathrm{ml}$ demonstrated the reverse effects on chondrocyte proliferation. Higher dosage of Palmvitee showed antiproliferative effect on cultured chondrocytes and thus, not suitable for chondrocyte monolayer expansion. This result was parallel with previous studies that the precursors of Palmvitee have an anti-proliferative effect on the growth of normal as well as cancerous cell lines $[3,4,5,21$, 22,25 - 27]. High dose treatment of $\alpha$-tocotrienols which is a subgroup of vitamin $E$ was said to induce anti-proliferative and apoptotic effects on normal mouse mammary epithelial cells at primary culture $[5,27]$. However, we are still unclear on the physiological significance of Palmvitee on human chondrocytes culture in this study. Palmvitee has been shown to interact in intracellular mechanisms pathways especially in the inhibition of the mitogenic-signaling pathways. This is done by inhibiting protein kinase $\mathrm{C}$, adynelate cyclase and cyclic AMP-dependent protein, thus induced the inhibition of cell mitogenic pathways [3, 4, 21, 22, 25, 26, 27]. Since most of the studies on understanding the mechanisms of Palmvitee in inducing anti-proliferative and apoptotic effects were done on the cancerous cells line, it is not known whether Palmvitee inhibit the human chondrocyte proliferation with the same mechanism. This needs to be clarified further through research [4, 21, 22, 25, 26, 27].

One step RT-PCR analysis on the freshly isolated chondrocytes showed only collagen type II expression with negative expression on collagen type I. This phenotype gene expression pattern specifies the characteristic of hyaline cartilage. Gene expression study results showed the addition of Palmvitee in the culture medium did not change the phenotype gene expression of cultured chondrocytes in multiple passages. All cultured chondrocytes showed a gradual reduction on collagen type II gene expression. This phenomenon was defined as cell dedifferentiation process and was due to flatten morphology of chondrocytes in monolayer culture $[12,13,29]$.

In cartilage reconstruction study, a large scale culture of human nasal septal chondrocytes in medium supplemented with or without $3 \mu \mathrm{g} / \mathrm{ml}$ Palmvitee exhibited dedifferentiation characteristic of negative expression on type II collagen gene. After 8 weeks of in vivo development, one step RT-PCR analysis on the total RNA extracted from the reconstructed cartilage showed a high re-expression of type II collagen. This showed that the addition of Palmvitee into the culture medium did not inhibit the re-differentiation process of chondrocyte in in vivo development. Histological evaluation on the reconstructed cartilage also demonstrated Palmvitee supplementation into the chondrocyte culture medium did not change the quality of the tissues after 8 weeks of in vivo development. Histological results showed the reconstructed cartilage were rich in cartilage proteoglycans matrix and exhibited a similar tissue organization comparable to the native cartilage. The reconstructed cartilage resemble more like a newly developed cartilage since it exhibited a higher cell density than the native cartilage.

\section{CONCLUSION}

Palmvitee at the concentration of $3 \mu \mathrm{g} / \mathrm{ml}$ in the human nasal septal chondrocytes culture medium provide beneficial value for chondrocytes monolayer expansion and cartilage reconstruction. Palmvitee at $3 \mu \mathrm{g} / \mathrm{ml}$ concentration increases chondrocyte growth rates, maintain the gene expression phenotype of cultured chondrocytes and reserve the quality of the reconstructed cartilage which resemble the quality of a native cartilage.

\section{ACKNOWLEDGMENTS}


We would like to thank Sultan Iskandar Foundation of Johor, Ministry of Science, Technology and Innovation, Malaysia for grants; IRPA 06-02-02-0037-EA189 and TOP DOWN BIOTEK 06-02-02-003 BTK/ER/022, Hospital Universiti Kebangsaan Malaysia and Faculty of Medicine, Universiti Kebangsaan Malaysia for making this study possible.

\section{REFERENCES}

1. Brigelius-Flohe, R. and Traber, M.G. (1999), Vitamin E: function and metabolism. FASEB J., vol. 13, (10), pp.1145-1155.

2. Yoshida, Y., Niki, E., and Noguchi, N. (2003), Comparative study on the action of tocopherols and tocotrienols as antioxidant: chemical and physical effects, Chem Phys Lipids, vol. 123, (1), pp. 63-75.

3. McIntyre, B.S, Briski, K.P., Gapor, A., and Sylvester, P.W. (2000), Antiproliferative and apoptotic effects of tocopherols and tocotrienols on preneoplastic and neoplastic mouse mammary epithelial cells, Proc Soc Exp Biol Med., vol. 224, (4), pp. 292-301.

4. Nesaretnam, K., Guthrie, N., Chambers, A.F., and Carroll, K.K. (1995), Effects of tocotrienols on the growth of a human breast cancer cell line in culture, Lipids., vol. 30, (12), pp. 1139-1143

5. McIntyre, B.S., Briski, K.P., Tirmenstein, M.A., Fariss, M.W., Abdul Gapor, and Sylvester, P.W. (2000), Antiproliferative and apoptotic effects of tocopherols and tocotrienols on normal mouse mammary epithelial cells, Lipids., vol. 35, (2), pp. 171-180.

6. Theriault, A., Chao, J.T., Wang, Q., Gapor, A., and Adeli, K. (1999), Tocotrienol: a review of its therapeutic potential, Clin Biochem., vol. 32, (5), pp. 309-319.

7. Sylvester, P.W., Nachnani, A., Shah, S., and Briski, K.P. (2002), Role of GTP-binding proteins in reversing the antiproliferative effects of tocotrienols in preneoplastic mammary epithelial cells, Asia Pac J Clin Nutr., vol. 11 (Suppl) 7, S452-S459.

8. Boscoboinik, D., Szewczyk, A., Hensey, C., and Azzi, A. (1991), Inhibition of cell proliferation by alpha-tocopherol, Role of protein kinase C. J. Biol Chem., vol. 266, (10), pp. 6188-6194.

9. Tasinato, A., Boscoboinik, D., Bartoli, G.M., Maroni P., and Azzi, A. (1995), d-alphatocopherol inhibition of vascular smooth muscle cell proliferation occurs at physiological concentrations, correlates with protein kinase $\mathrm{C}$ inhibition, and is independent of its antioxidant properties, Proc Natl Acad Sci USA, vol. 92, (26), pp. 12190-12194.

10. Marler, J.J., Upton, J., Langer, R., and Vacanti, J.P. (1998), Transplantation of cells in matrices for tissue regeneration, Adv Drug Deliv Rev., vol. 33, pp. 165-182.

11. Vacanti, C.A., Page, K.T., Woo, S.K., Sakata. J., Upton, J., and Vacanti, J.P. (1994), Experimental tracheal replacement using tissue-engineered cartilage, J Pediatr Surg, vol. 29 (2), pp. 201-205.

12. Vacanti, J. P. and Langer, R. (1999), Tissue engineering: the design and fabrication of living replacement devices for surgical reconstruction and transplantation, Lancet, vol. 354 (suppl I), pp. 32-34.

13. Rotter, N., Bonassar, L.J., Tobias, G., Lebl, M., Roy, A.K., and Vacanti, C.A. (2001), Age dependence of cellular properties of human septal cartilage: implications for tissue engineering, Arch Otolaryngol Head Neck Surg., vol. 127, p. 1248.

14. Vetter, U., Heit, W., Helbing, G., Heinze, E., and Pirsig, W. (1984), Growth of the human septal cartilage: cell density and colony formation of septal chondrocytes, Laryngoscope, vol. 94 (9), pp. 1226-1229. 
15. Okasaki, R., Sakai, A., Nakamura, T., Kunugita, N., Norimura, T., and Suzuki, K. (1996), Effects of transforming growth factor betas and basic fibroblast growth factor on articular chondrocytes obtained from immobilized rabbit knees (dagger), Ann Rheum Dis, vol. 55 (3), pp. 181-186.

16. Klaus, V.D.M., Verena, G., Helga, V.D.M., and Peter, M. (1997), Relationship between cell shape and type of collagen synthesized as chondrocytes lose their cartilage phenotype in culture, Nature, vol. 267, pp. 531-532.

17. Yang, S.Y., Ahn, S.T., Rhie, J.W., Lee, K.Y., Choi, J.H., Lee, B.J., and Oh, G.T. (2000), Platelet supernatant promotes proliferation of auricular chondrocytes and formation of chondrocyte mass, Ann Plast Surg, vol. 44 (4), pp. 405-411.

18. Chomczynski, P.A, (1993), Reagent for the single-step simultaneous isolation of RNA, DNA and proteins from cell and tissue samples, Biotechniques, vol. 15 (3), pp. 532-534.

19. Chomczynski, P. and Sacchi, N. (1987), Single step method of RNA isolation by acid guanidinium thiocyanate-phenol-chloroform extraction, Anal Biochem, vol. 162 (1), pp. 156-159.

20. Jamila, M., Al-S., Jiwan, S.S., Suad, N. Al-H., Hanan, A. Al-A., Amani, Al-O., Latifa, Al-H., Nissar, A., Isa, B.M., and Johari, M. (2004), Developing functional foods using red palm olein. IV: Tocopherols and tocotrienols, Food Chem., vol. 85, pp. 579-583.

21. Campbell, S., Stone, W., Whaley, S., and Krishnan, K. (2003), Development of gamma $(\gamma)$-tocopherol as a colorectal cancer chemopreventive agent, Crit Rev Oncol Hematol, vol. 47 (3), pp. 249-259.

22. Takahashi, K. and Loo, G. (2004), Disruption of mitochondria during tocotrienolinduced apoptosis in MDA-MB-231 human breast cancer cells, Biochem Pharmacol, vol. 67 (2), pp. 315-324.

23. Setiadi, D.H., Chass, G.A., Koo, J.C.P., Penked, B., and Csizmadia, I.G. (2003), Exploratory study on the full conformation space of $\alpha$-tocopherol and its selected congeners, J. Mol Struct, pp. 666-667; pp. 439-443.

24. Landes, N., Birringer, M., and Brigelius-Flohe R. (2003), Homologous metabolic and gene activating routes for vitamins E and K. Mol Aspects Med., vol. 24, pp. 337-44.

25. Kausar, H., Bhasin, G., Zargar, M.A., and Athar, M. (2003), Palm oil alleviates 12-Otetradecanoyl-phorbol-13-acetate-induced tumor promotion response in murine skin, Cancer Lett., vol. 192 (2), pp. 151-160.

26. Galli, F., Stabile. A.M., Betti, M., Conte, C., Pistilli, A., Rende, M., Floridi, A., and Azzi, A. (2004), The effect of $\alpha$ - and $\gamma$-tocopherol and their carboxyethyl hydroxychroman metabolites on prostate cancer cell proliferation, Arch Biochem and Biophys., vol. 423 (1), pp. 97-102.

27. Azzi, A. and Stocker, A. (2000), Vitamin E: non-antioxidant roles, Prog Lipid Res., vol. 39 (3), pp. 231-255.

28. Loty, S., Sautier, J.M., and Forest, N. (2000), Phenotypic modulation of nasal septal chondrocytes by cytoskeleton modification, Biorheology, vol. 37 (1-2), pp. 117-125.

29. Homicz, M.R., Schumacher, B.L., Sah, R., and Watson, D. (2002), Effects of serial expansion of septal chondrocytes on tissue-engineered neocartilage composition, Otolaryngol Head Neck Surg., vol. 127 (5), pp. 398-408. 